\title{
A Systematic Review and Meta-Analysis of Studies Examining the Use of Brain Laser Interstitial Thermal Therapy versus Craniotomy for the Treatment of High-Grade Tumors in or near Areas of Eloquence: An Examination of the Extent of Resection and Major Complication Rates Associated with Each Type of Surgery
}

\author{
Gene H. Barnett ${ }^{a-c}$ Jeffrey D. Voigt ${ }^{f}$ Manmeet S. Alhuwaliad, e \\ ${ }^{a}$ Cleveland Clinic Lerner College of Medicine, Case Western Reserve University, ${ }^{b}$ Rose Ella Burkhardt Brain Tumor and Neuro-Oncology

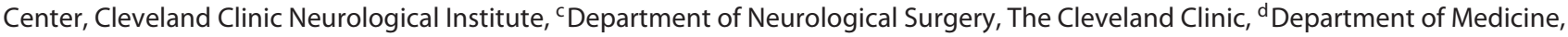 \\ Cleveland Clinic Lerner College of Medicine, Case Western Reserve University, and 'Brain Metastasis Research Program, Clinical Trials, \\ Operations, The Rose Ella Burkhardt Brain Tumor and Neuro-Oncology Center, Neurological Institute, Cleveland, Ohio, and ${ }^{\mathrm{f} M e d i c a l}$ \\ Device Consultants of Ridgewood, LLC, Ridgewood, N.J., USA
}

\section{Key Words}

Brain laser interstitial thermal therapy - Areas of eloquence Complications · Extent of resection

\begin{abstract}
Background: The extent of resection (EOR) of high-grade gliomas (WHO grade III or IV) in or near areas of eloquence is associated with overall patient survival, but with higher major neurocognitive complications. Methods: A systematic review and meta-analysis was undertaken of the peer-reviewed literature in order to identify studies which examined EOR or extent of ablation (EOA) and major complications (defined as neurocognitive or functional complications which last $>3$ months duration after surgery) associated with either brain laser interstitial thermal therapy (LITT) or open craniotomy in high-grade tumors in or near areas of eloquence. Results: Eight studies on brain LITT ( $n=79$ patients) and 12 craniotomy studies ( $\mathrm{n}=1,036$ patients) were identified which examined either/both EOR/EOA and complications. Meta-analysis demonstrated an EOA/EOR of $85.4 \pm$
\end{abstract}

$10.6 \%$ with brain LITT versus $77.0 \pm 40 \%$ with craniotomy (mean difference: $8 \%$; 95\% Cl: 2-15; $\mathrm{p}=0.01$; inverse variance, random effects model). Meta-analysis of proportions of major complications for each individual therapy demonstrated major complications of $5.7 \%(95 \% \mathrm{Cl}: 1.8-11.6)$ and 13.8\% (95\% Cl: 10.3-17.9) for LITT and craniotomy, respectively. Conclusion: In patients presenting with high-grade gliomas in or near areas of eloquence, early results demonstrate that brain LITT may be a viable surgical alternative.

(c) 2016 The Author(s)
Published by S. Karger AG, Basel

\section{Introduction}

In the United States, approximately $50 \%$ of the over 23,000 central nervous system tumors are classified as being at high risk for neurological complications during resection $[1,2]$. The main reasons for the high-risk nature of these resections are due to the tumor's location in or near areas of eloquence or in their deep-seated nature within the brain. In these types of brain tumors, extent of

\begin{tabular}{|c|c|c|}
\hline KARGER & $\begin{array}{l}\text { () } 2016 \text { The Author(s) } \\
\text { Published by S. Karger AG, Basel } \\
1011-6125 / 16 / 0943-0164 \$ 39.50 / 0\end{array}$ & $\begin{array}{l}\text { Karger } \\
\text { Open access }\end{array}$ \\
\hline www.karger & $\begin{array}{l}\text { This article is licensed under the Creative } \\
\text { NonCommercial-NoDerivatives } 4.0 \text { Intern } \\
\text { NC-ND) (http://www.karger.com/Service } \\
\text { Usage and distribution for commercial pur } \\
\text { tribution of modified material requires writ }\end{array}$ & $\begin{array}{l}\text { Commons Attribution- } \\
\text { tional License (CC BY- } \\
\text { s/OpenAccessLicense). } \\
\text { poses as well as any dis- } \\
\text { ten permission. }\end{array}$ \\
\hline
\end{tabular}

Jeffrey D. Voigt

99 Glenwood Rd

Ridgewood, NJ 07450 (USA)

E-Mail jdv4957@aol.com 
resection (EOR) (cytoreductive debulking) via craniotomy can be compromised and major neurocognitive complications (i.e. those complications extending $>3$ months after surgery) associated with resection are higher (versus tumors that are not near areas of eloquence). Both of these factors are associated with compromised function after surgery and survival [3-8]. Further, EOR and surgical complications may also reduce the performance status of the patient and the ability to receive adjuvant therapy that can affect survival $[9,10]$.

As it relates to EOR, the published literature commonly shows that the complication rates with open craniotomy are $15 \%$ or greater $[9,11,12]$ in patients with highgrade gliomas located in or near areas of eloquence. Further, craniotomy when used in these tumors commonly results in suboptimal resection, thereby compromising overall survival. In published studies, the EOR has been found to improve survival at $\geq 78 \%$; including a stepwise improvement in survival when examined for EORs at $\geq 80, \geq 90$, and $100 \%$ [5]. The current National Comprehensive Cancer Network (NCCN) recommendation for resection of high-grade gliomas, where maximal safe resection is not feasible, is to perform stereotactic biopsy, open biopsy, or subtotal resection [13]. These surgical options, however, do not include any category of evidence and consensus recommendation from the NCCN.

It has also been noted that only in 1 in 4 patients with recurrent glioblastoma (recurrent high-grade glioma) is repeat surgery considered, and repeat surgery is not recommended for patients with involvement of prespecified eloquent/critical brain regions [14]. Additionally, factors that predict survival after surgery for recurrent high-grade glioma include preoperative Karnofsky performance status, extent of surgical resection, and age [14].

Recently, brain laser interstitial thermal therapy (LITT) has been utilized as an alternative to open craniotomy. LITT may confer an advantage in EOR (referred to as extent of ablation with brain LITT, or EOA) along with lower complication rates in patients presenting with high-grade gliomas; however, it has not been examined in a systematic fashion. For this reason, a systematic review of the literature and meta-analysis was undertaken in order to evaluate the EOR/EOA and complication rates of brain LITT compared to open craniotomy.

\section{Methods}

A systematic review of the literature was undertaken on April 28, 2015 using the methodology described below.
Electronic Searches

The following electronic databases were searched on April 28, 2015:

- The Cochrane Collaboration

- The Cochrane Central Register of Controlled Trials (CENTRAL; 2015, Issue 3)

- The Database of Abstracts of Reviews of Effects (DARE; 2015, Issue 1)

- The NHS Economic Evaluation Database (NHS EED; 2014, Issue 1)

- PubMed

The above sources were searched using the following keywords: 'high grade AND glioma AND resect* AND outcome'. In addition, ClinicalTrials.gov was searched on April 28, 2015 to identify any trials in process or which had been recently completed using the same search terms as above. Lastly, Google was searched on April 28, 2015 using the search terms: 'high grade AND glioma AND eloquence AND resection AND outcome. The first 4 pages of hits were evaluated.

\section{Searching Other Resources}

The following journals were also hand searched:

- Neurosurgery (most recent 12 months up to April 28, 2015; searched on April 28, 2015)

- Journal of Neurosurgery (most recent 6 months up to April 28, 2015; searched on April 28, 2015)

- Journal of Neuro-Oncology (most recent 6 months up to April 28, 2015; searched on April 28, 2015)

- Journal Clinical Oncology (most recent 6 months up to April 28, 2015; searched on April 28, 2015).

Hand searching the journals above was undertaken because of the time lag between their publication and availability on electronic indexes.

Websites for both Monteris Medical and Medtronic were searched for clinical studies associated with the NeuroBlate (Monteris) and Visualase (Medtronic) on April 29, 2015. These technologies are currently on the market and are being used to treat patients with high-grade glioma/recurrent high-grade glioma.

Data Collection and Analysis

PRISMA guidelines [15] were followed using the PRISMA 2009 checklist.

\section{Selection of Studies and Data Used in Analysis}

Studies reporting on patients who received either open craniotomy or the brain LITT procedure for treating high-grade primary or recurrent brain tumors (WHO grade III or IV) in or near areas of eloquence and/or of a deep-seated nature (e.g. brain stem) were used. Studies had to explicitly state that the tumor was in or near an area of eloquence in order to include them in this analysis.

\section{Data Extraction and Management}

A data extraction form was developed (see online supplementary Appendix 1; for all online supplementary material, see www. karger.com/doi/10.1159/000446247). One review author extracted the data using this form (J.D.V.). A second (G.H.B.) and third author (M.S.A.), reviewed and validated the data. If a study had more than one publication, all versions were considered in order to maximize data extraction, and the primary publication was identified along with the secondary references. Online supplemen- 


\section{PRISMA Flow Diagram}
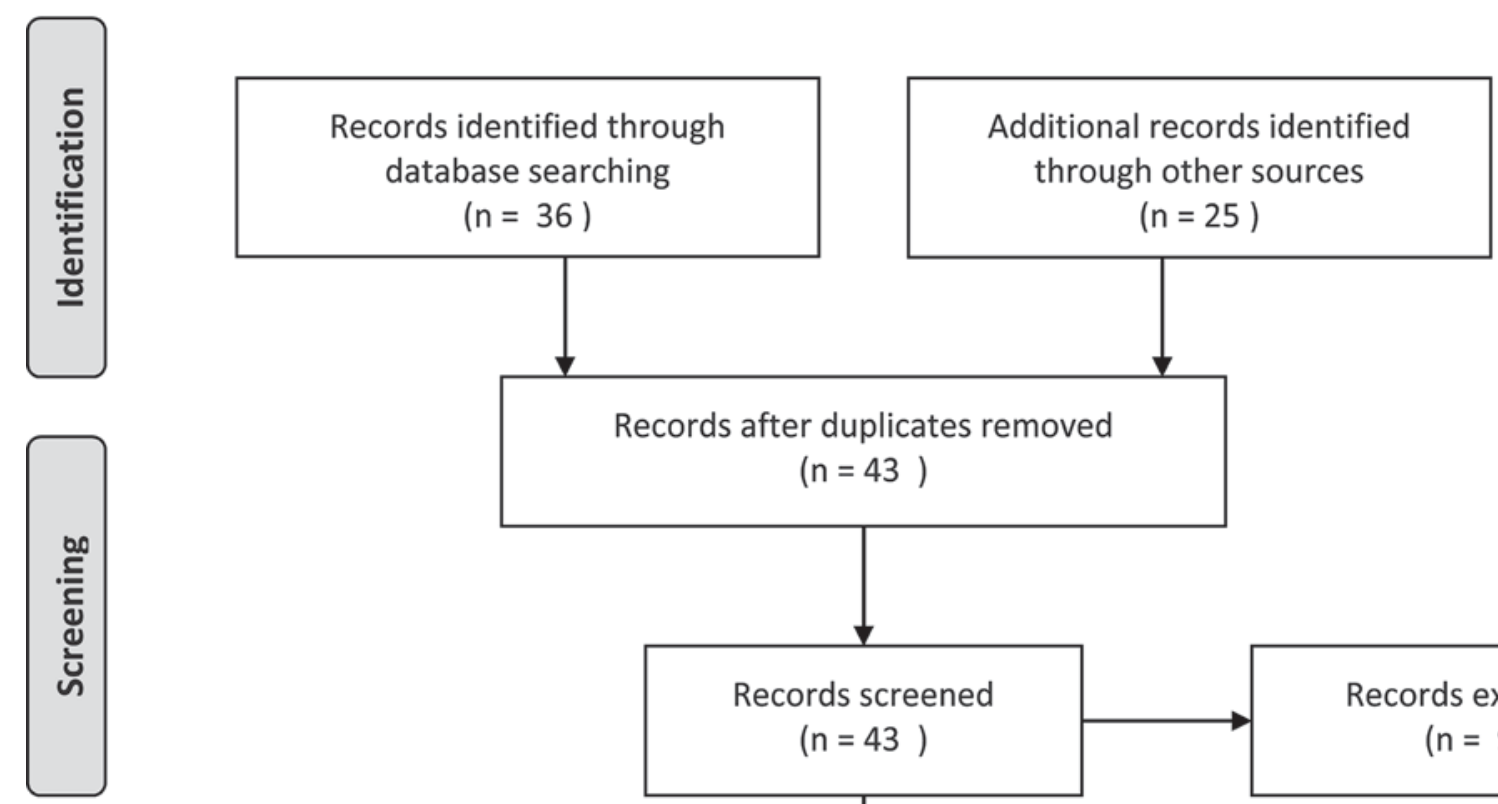

Records after duplicates removed

$$
\text { ( } n=43 \text { ) }
$$
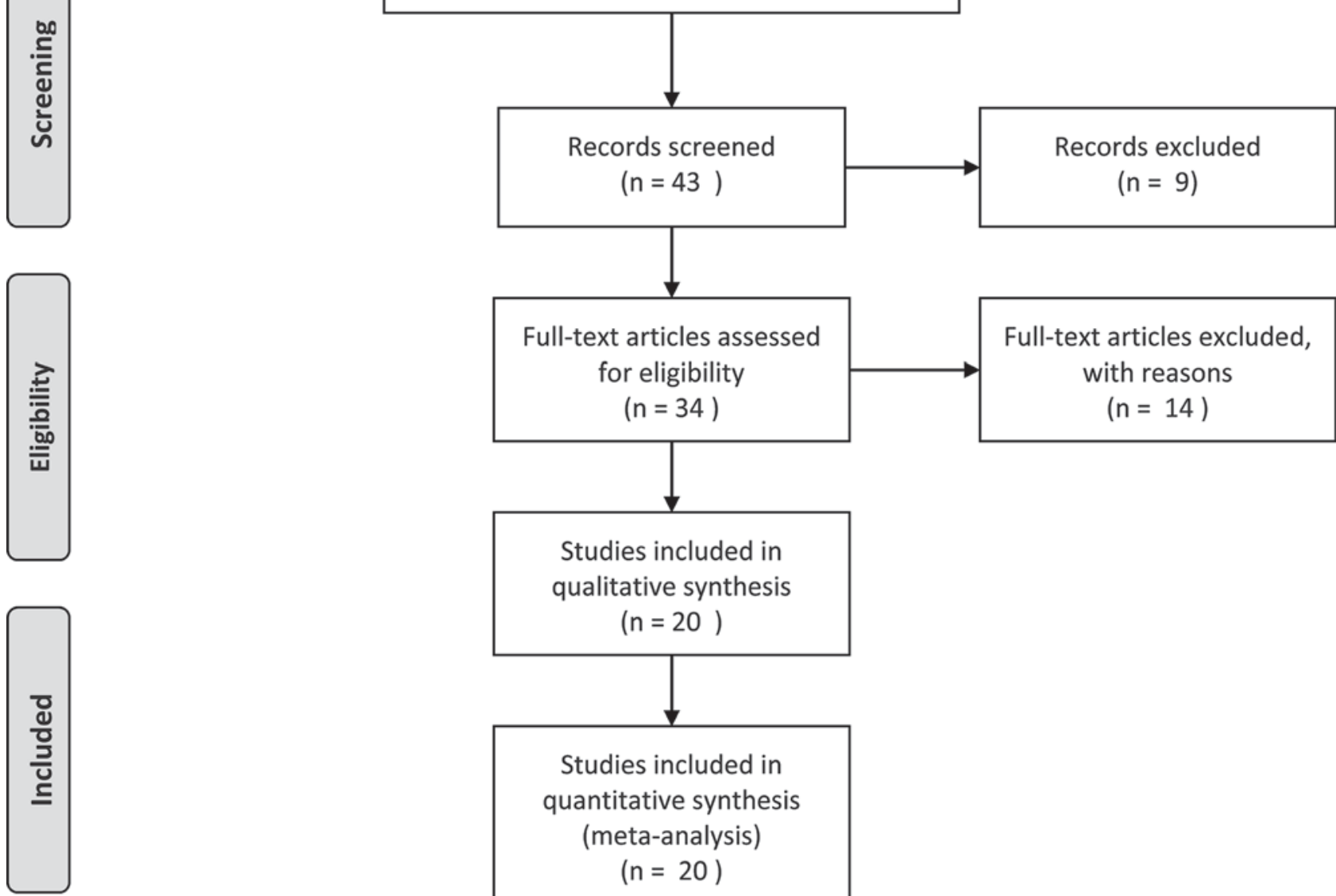

Studies included in qualitative synthesis $(n=20)$

Studies included in quantitative synthesis

(meta-analysis)

$$
(n=20)
$$

From: Moher D, Liberati A, Tetzlaff J, Altman DG, The PRISMA Group (2009). Preferred Reporting /tems for Systematic Reviews and MetaAnalyses: The PRISMA Statement. PLoS Med 6(6): e1000097. doi:10.1371/journal.pmed1000097

For more information, visit www.prisma-statement.org.

Fig. 1. PRISMA flow diagram. 
Table 1. Patient baseline characteristics

\begin{tabular}{lcll}
\hline & LITT & Craniotomy & $\mathrm{p}$ \\
\hline Mean age \pm SD, years & $54.3 \pm 10.81$ & $45.6 \pm 14.81$ & $<0.00001$ \\
M/F, n & $43 / 24$ & $307 / 215$ & \\
Males/total pts, n & $43 / 67$ & $307 / 522$ & 0.37 \\
Karnofsky score (preop) & $73.4 \pm 10$ & $78.4 \pm 7.95$ & 0.0006 \\
Recurrent glioma, n & 51 & 198 & \\
Primary glioma, n & 24 & 699 & \\
Recurrent/all gliomas ratio & $68 \%$ & $22 \%$ & $<0.00001$ \\
\hline
\end{tabular}

Values represent means \pm SD unless otherwise indicated. $p t s=$ Patients; preop $=$ preoperative.

tary Appendix 1 shows the data collection form utilized and as well definitions of the risk of bias assessments used. Risk of bias included: random sequence generation, allocation concealment, blinding (patients, clinicians performing procedure, clinical assessors), incomplete data reporting, selective reporting, and other bias (e.g. funding, consulting, etc.).

\section{Measures of Treatment Effect}

Analysis of the studies included was accomplished first by evaluating the mode of treatment (studies combined for LITT and studies combined for craniotomy) using meta-analysis of proportions (for complications; both random and fixed effects) using MedCalc version 15.6.1 (MedCalc Software, Mariakerk, Belgium). In comparing results of binary outcomes, these treatment comparisons were presented as risk differences with corresponding 95\% CI. For continuous data (e.g. EOR), the mean difference was utilized (Cochrane Review Manager 5.3, statistical package). The results of the treatment effect(s) are reported in Results. Heterogeneity was evaluated using the $\mathrm{I}^{2}$ statistic.

\section{Conversion of Median, Range to Mean and Standard}

Deviation

Conversion to a mean and standard deviation in studies where median and range only existed was accomplished using methodology as per Hozo at al. [16].

\section{Results}

There were 15 studies identified on brain LITT [1731]. Seven studies were excluded for the following reasons: inability to identify whether the tumors were located in an area(s) of eloquence (2 studies) [17, 19], tumors were not located in areas of eloquence (1 study) [21], tumors were metastasized from another part of the body ( 3 studies) $[23,24,28]$, and one [31] because it was a duplicate of studies/patients included in the analysis [30]. These brain LITT studies included along with their rele- vant findings can be found in online supplementary Appendix 2.

There were 19 studies identified using craniotomy [3, $4,7,9-12,32-43]$. Of these, 6 articles were excluded [3, $32,37,42,43$ ] due to an inability to break out complications related to surgery in areas of eloquence. Additionally, one article was excluded [12] due to the assumption that these patients were reported on in Kim et al. [33] and to reduce the chance of double counting. Online supplementary Appendix 2 shows the craniotomy studies included (total of 12 craniotomy studies included). Thus, a total of 20 articles ( 8 brain LITT +12 craniotomy) were used in the meta-analysis evaluating EOR and complication rates. Online supplementary Appendix 2 also shows all LITT and craniotomy studies that were excluded and the reasons why. A PRISMA flow diagram showing the number of studies identified can be found in figure 1 .

\section{Patient Demographics}

Table 1 reviews the summary demographics of the patients included in the analysis for both brain LITT and craniotomy. It can be seen in the data (where reported on and identified) that patients undergoing brain LITT (vs. craniotomy) were older (54.3 vs. 45.6 years), had lower Karnofsky preoperative scores (73.4 vs. 78.4 ), and that LITT was used more frequently in recurrent high-grade versus primary gliomas $[68 \%(51 / 75)$ versus $22 \%$ $(198 / 897)]$.

\section{Neurocognitive/Functional Complication Rates (>3 Months Duration)}

There were 8 brain LITT studies totaling 77 patients included in this analysis $[18,20,22,24,26,27,29,30]$. It was found that 3 such complications occurred in these studies (table 2). There were 11 craniotomy studies totaling 1,036 patients included in this analysis $[3,9-12,34-36,39-41]$. There were 141 such complications that occurred (table 2). These data were examined by treatment type. The proportion of major complications was $5.7 \%$ for brain LITT [95\% CI: 1.8-11.6; $\mathrm{I}^{2}=0 \%$; random effects (RE) model; fig. 2] and $13.9 \%$ for craniotomy (95\% CI: 10.3-17.9; $\mathrm{I}^{2}=65 \%$; RE model; fig. 3) (for the statistics, see online suppl. Appendix 3). In a combined comparison of brain LITT versus craniotomy, a $10 \%$ reduction in the absolute risk difference, favoring brain LITT (risk difference $=-0.10$; 95\% CI: -0.15 to $-0.05 ; \mathrm{p}<0.0001$; Mantel-Haenszel RE model) was identified. 
Table 2. Major neurocognitive complications ( $>3$ months duration) seen in patients with high-grade glioma treated with LITT and craniotomy

\begin{tabular}{|c|c|c|c|c|c|}
\hline & \multicolumn{2}{|l|}{ LITT } & \multicolumn{2}{|c|}{ Craniotomy } & \multirow[t]{2}{*}{ Primary/recurrent } \\
\hline & comps, $\mathrm{n}$ & total pts & comps, $\mathrm{n}$ & total pts & \\
\hline Carpentier [25], 2012 & 0 & 4 & & & recurrent \\
\hline Chaichana [39], 2014 & & & 34 & 259 & primary \\
\hline Gulati [9], 2011 & & & 22 & 141 & primary \\
\hline Hundsberger [40], 2014 & & & 2 & 13 & primary \\
\hline Jethwa [26], 2012 & 0 & 7 & & & recurrent \\
\hline $\operatorname{Kim}[33], 2009$ & & & 22 & 100 & primary/recurrent \\
\hline Konglund [10], 2013 & & & 6 & 47 & primary \\
\hline Kreig [36], 2013 & & & 4 & 47 & primary \\
\hline Kuhnt [35], 2011 & & & 7 & 79 & primary \\
\hline Mohammadi [30], 2014 & 2 & 35 & & & primary/recurrent \\
\hline Reimer [20], 1998 & 0 & 4 & & & recurrent \\
\hline Sakai [18], 1992 & 0 & 3 & & & primary \\
\hline Sanai [34], 2010 & & & 10 & 104 & primary/recurrent \\
\hline Satoer [38], 2012 & & & 11 & 25 & primary/recurrent \\
\hline Sawaya [11], 1998 & & & 20 & 154 & primary/recurrent \\
\hline Schucht [41], 2014 & & & 3 & 67 & primary \\
\hline Schwarzmaier [22], 2006 & 0 & 16 & & & recurrent \\
\hline Sloan [27], 2013 & 1 & 8 & & & recurrent \\
\hline Total & 3 & 77 & 141 & 1,036 & \\
\hline
\end{tabular}

comps = Complications; pts = patients.

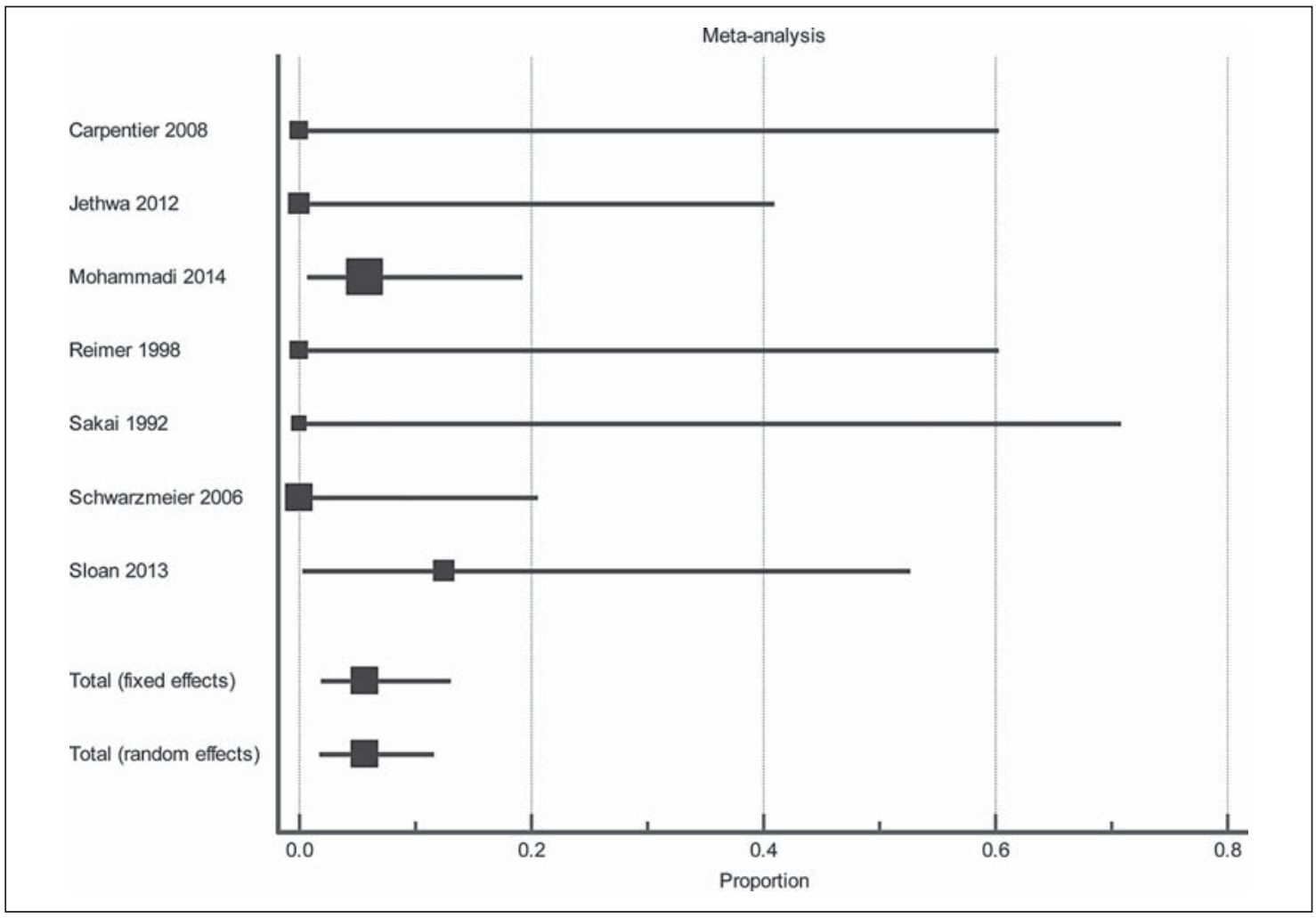

Fig. 2. Meta-analysis: proportion major complications LITT. 
Table 3. EOR/EOA

\begin{tabular}{|c|c|c|c|c|c|c|}
\hline Study & Total pts & Mean EOR & SD EOR & Total pts & Mean EOR & SD EOR \\
\hline Carpentier [25], 2012 & 4 & $100.00 \%$ & $0.00 \%$ & & & \\
\hline Jethwa [26], 2012 & 3 & $100.00 \%$ & $0.00 \%$ & & & \\
\hline Kuhnt [35], 2011 & & & & 79 & $77.00 \%$ & $75.00 \%$ \\
\hline Mohammadi [30], 2014 & 35 & $82.50 \%$ & $16.50 \%$ & & $0.00 \%$ & $0.00 \%$ \\
\hline Orringer [7], 2012 & & & & 18 & $84.00 \%$ & $18.00 \%$ \\
\hline Sakai [18], 1992 & 3 & $100.00 \%$ & $0.00 \%$ & & & \\
\hline Sanai [34], 2010 & & & & 40 & $77.25 \%$ & $13.25 \%$ \\
\hline Satoer [38], 2012 & & & & 25 & $70.70 \%$ & $24.25 \%$ \\
\hline Schroeder [29], 2014 & 3 & $89.00 \%$ & $16.00 \%$ & & & \\
\hline Sloan [27], 2013 & 8 & $78.00 \%$ & $12.00 \%$ & & & \\
\hline Total number of pts & 56 & & & 162 & & \\
\hline Averages & & $85.40 \%$ & $10.60 \%$ & & $77.00 \%$ & $40.00 \%$ \\
\hline
\end{tabular}

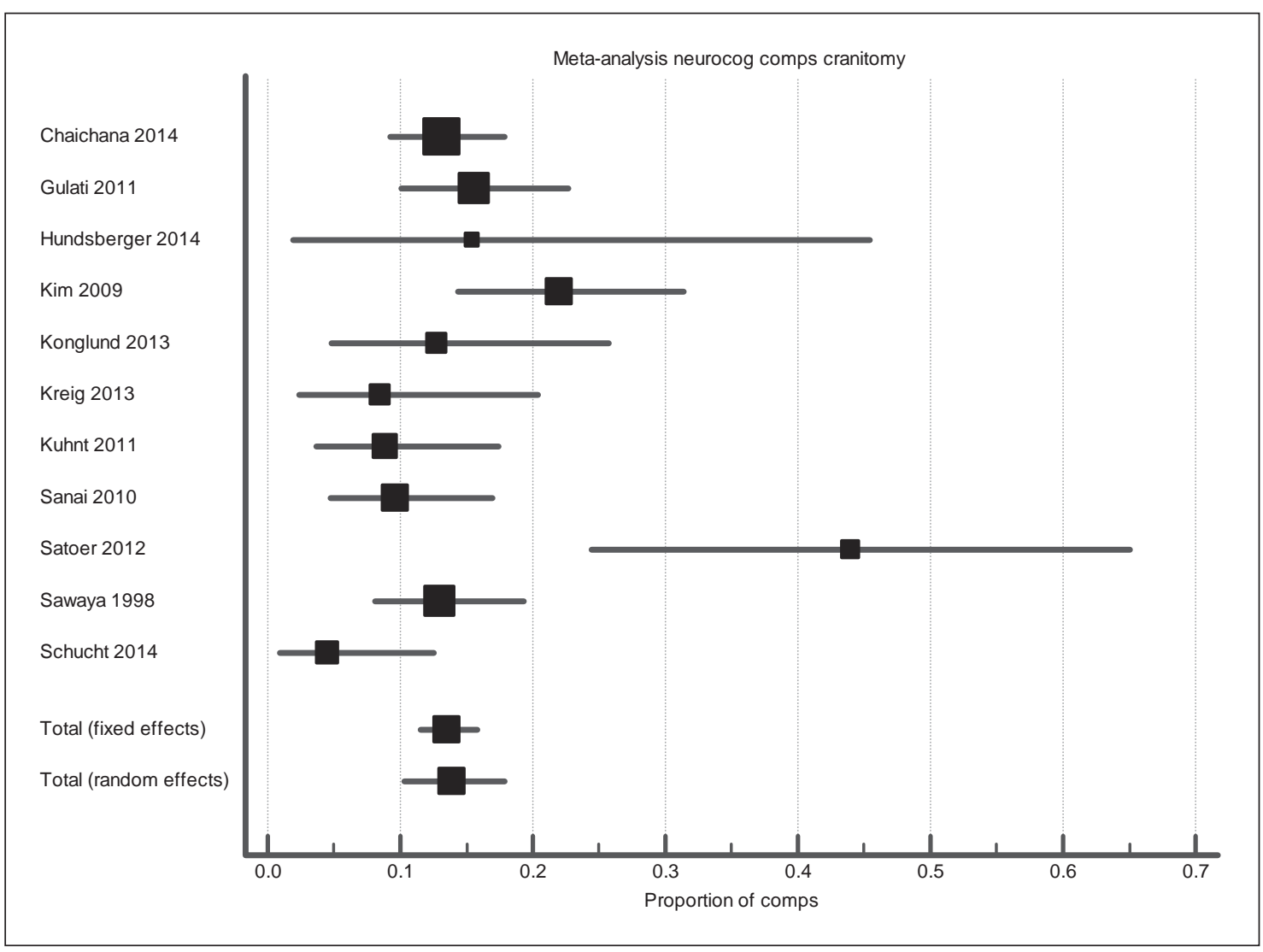

Fig. 3. Meta-analysis: proportion major complications craniotomy. 


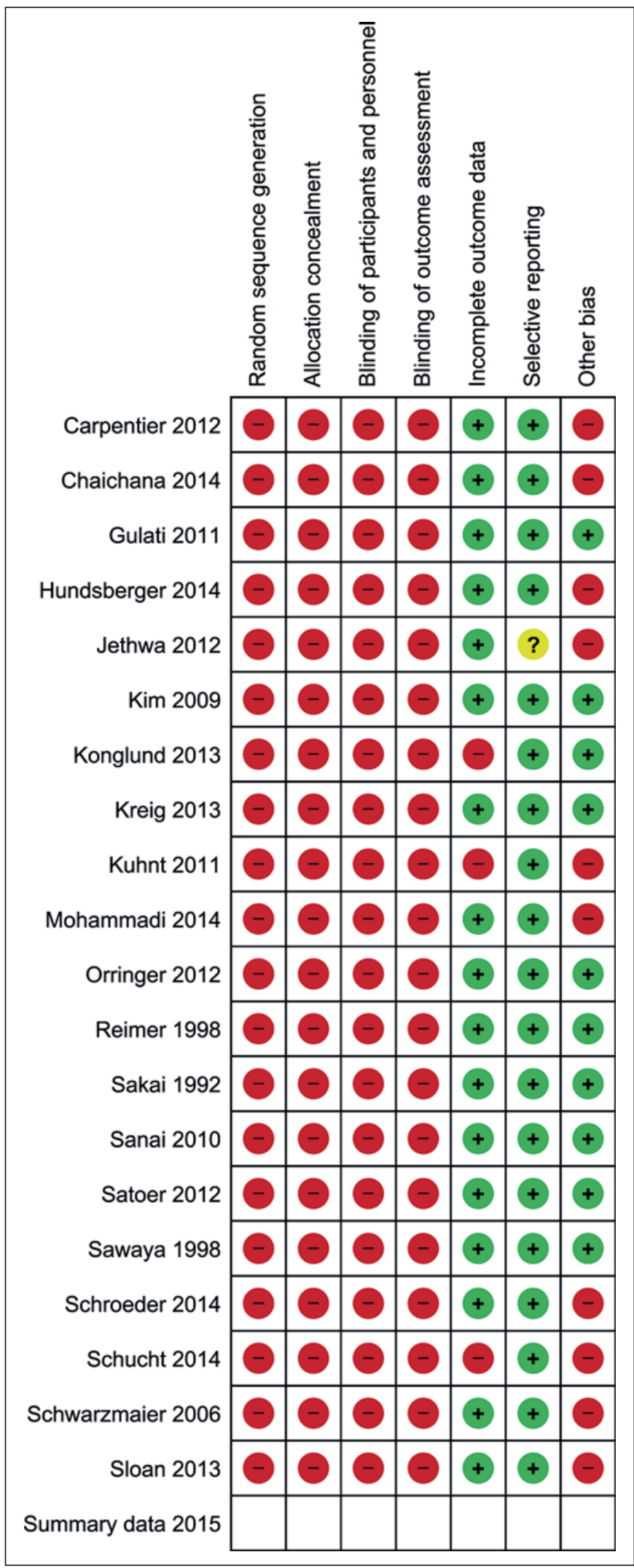

Fig. 4. Risk of bias summary.

\section{Extent of Resection}

There were 6 brain LITT studies totaling 56 patients included in this analysis $[18,24,26,27,29,30]$. The weighted mean \pm standard deviation for EOR/EOA for brain LITT was calculated to be $85.4 \pm 10.6 \%$ (table 3 ). There were 4 craniotomy studies totaling 162 patients included in this analysis $[5,7,35,38]$. The weighted mean \pm standard deviation EOR for craniotomy was calculated to be $77.0 \pm 40 \%$ (table 3 ). The data were also combined and a comparison made between the two groups. The mean difference between the two groups was $8 \%$ (mean difference $=0.08 ; 95 \%$ CI: $0.02-0.15 ; \mathrm{p}=0.01$; inverse variance $\mathrm{RE}$ model), favoring an improved EOR with brain LITT.

\section{Discussion}

The goal of surgery in high-grade gliomas is to achieve maximal resection (cytoreductive debulking) with minimal neurocognitive complications [44]. This objective is also important in order to gain maximal benefit from adjunct therapies. Overall survival can be improved when these occur $[8,9]$. In this analysis, a study of the early published results of LITT in high-grade gliomas, a statistically significant improvement in EOR/EOA along with a reduction in major neurocognitive complications appears to be possible with LITT versus craniotomy. The EOR rates in the craniotomy arm of this analysis is consistent with EOR meta-analyses examining the use of intraoperative stimulation brain mapping in tumors located in areas of eloquence [45]. Further, a reduction in these types of complications may have a positive effect in reducing the incidence and cost of follow-on care for these types of patients (e.g. lower discharge rate to a skilled nursing facility). There was minimal overlap in the confidence intervals shown on the proportion of complications. What this may infer is that the complication rates between the two groups are not statistically different. This is a hypothesis that should be tested in a well-designed trial that compares the use of resection versus LITT in tumors in or near areas of eloquence. It also appears that based on the findings in the literature, this improved EOR/EOA with lower complications may confer an overall (clinical) survival benefit along with an ability to optimize the use of adjunct therapies [5]. However, again this needs to be tested in a well-designed trial.

In this analysis, the patients treated with LITT were older and had lower Karnofsky Performance Index scores. This is a notable finding in that clinicians are re- 
Fig. 5. Risk of bias graph.

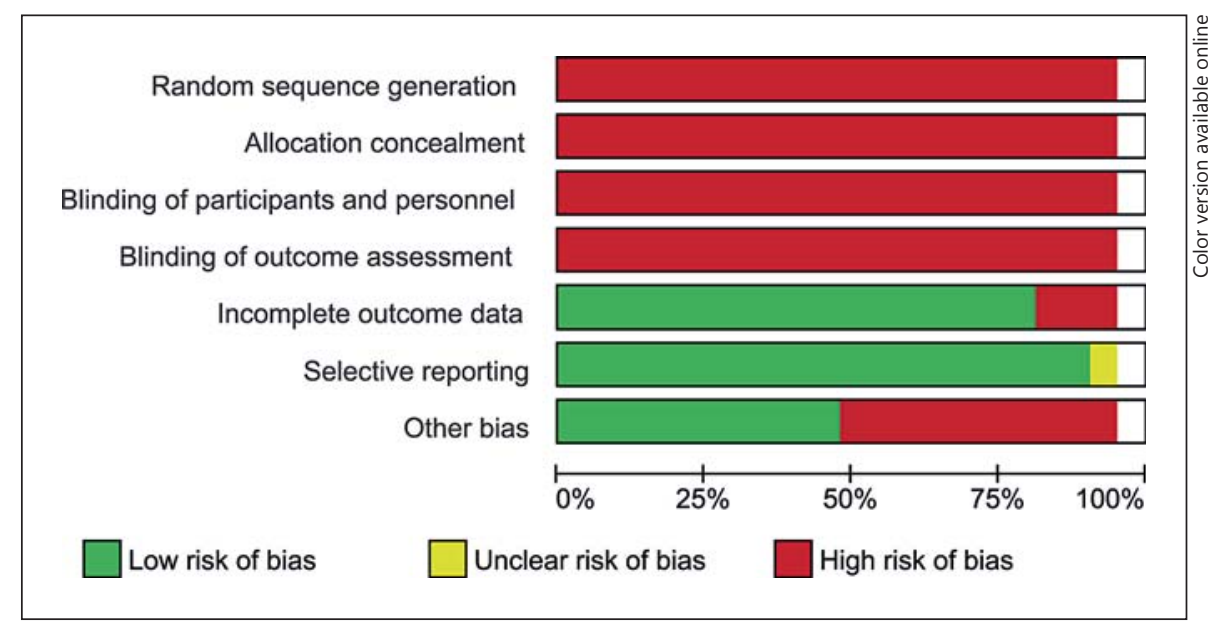

porting mainly on brain LITTs used in treating recurrent high grade gliomas. As mentioned above, recurrent gliomas are an indication that appears not to have any reasonable surgical alternatives - especially for tumors located in areas of eloquence [46]. Thus, it is reaffirming to find these types of positive results (i.e. results 'comparable' to the use of craniotomy in primary high-grade gliomas) in extremely challenging patients with the use of brain LITT.

Further, the types of tumors where brain LITT is being used currently have no clinical or evidence recommendations for optimal resection. In fact, the NCCN guidelines noted above suggest the use of subtotal resection either with open craniotomy or with biopsy (without any evidentiary recommendation) in these types of patients [13]. This analysis supports the consideration for inclusion in treatment guidelines of brain LITT as an option for patients with high-grade gliomas in or near areas of eloquence.

Based on the high heterogeneity of the data, which may be due to the different conditions of the patients (recurrent versus primary high-grade gliomas, Karnofsky score, age), it may make combining the data for metaanalysis purposes questionable. It was also not possible to break out EOR and complications in recurrent highgrade gliomas for patients undergoing craniotomy in the studies identified (the studies did not go into this level of detail); therefore, an apples-to-apples comparison of brain LITT versus craniotomy could not be made. However, the recommendation being made based on the meta-analysis findings that brain LITT should be considered in recurrent high-grade glioma therapy based on improved EOR and lower complications may be reasonable, considering there really is no surgically recommended option [46].

There are several limitations to our analysis. First, the assumption made is that EOR is equal to EOA. Mohammadi et al. [30] suggest that the cytoreductive effect of hyperthermia can be considered as equivalent to surgical debulking (or EOR). This analysis makes the same assumption as the heated (via LITT) tumor tissue appears necrotic when viewed on MRI. Other studies have stated there likely will never be randomized trials comparing resection to any kind of ablation in tumors, but do state that ablation represents an effective treatment option for those unsuitable to conventional surgical treatment [47], which is the case with the vast majority of these LITT patients reported on herein. Furthermore, a meta-analysis which compares hepatic resection to radiofrequency ablation shows equivalency of efficacy with tumors of $<2 \mathrm{~cm}$ in diameter [48]. The brain tumors reported on in this study (approx. 35\% of them) were of this size. Unfortunately, such tumors could not be broken out in evaluating complications or EOR. Second, these findings should be interpreted within the limitations inherent in combining observational studies for meta-analytic purposes as the data may be confounded by a number of factors including patient selection, patient condition, and the fact that these patients are treated at academic medical centers. Third, the data evaluated are not from high-quality studies [high quality defined with identified randomization, allocation concealment, blinding (including patient and outcome assessors), complete outcome data reported on], as these types of studies likely do not exist (fig. 4, 5). It may, however, be difficult to randomize patients with this aggressive disease which ultimately results in death. Fourth, the 
EOR/EOA results identified for brain LITT may be understated due to the fact that one of the included studies was a first-in-man study of brain LITT [27]. There were restrictions placed on this trial which likely limited the $\mathrm{EOR} / \mathrm{EOA}$ to $78 \%$ (i.e. allowance for only one pass of the laser within the tumor; amount of energy employed). Fifth, there is a fair amount of data omitted on EOR and in patient characteristics in the trials identified. However, the EORs and complications identified and used in the summary statistics above are consistent with the types of patients reported on in the literature for both EOR and complication rates [45]. Lastly, the cumulative number of patients reported on in the literature with brain LITT is relatively small. However, there is also a learning curve associated with brain LITT and these results may likely improve over time as demonstrated in prior studies [22]. Additionally, improved guidance of brain LITT under MRI may also provide for improved outcomes related to complications and EOR/EOA. Earlier studies included in this analysis did not use MRI guidance and thus the overall effect (on EOR/EOA and on complications) of brain LITT + MRI guidance (the current technology) may be underestimated.

\section{Conclusions}

Brain LITT may demonstrate an improvement in EOR/EOA and may reduce major neurocognitive complications compared to open craniotomy in patients with high-grade gliomas. Both of these outcomes impact overall survival. Clinical guidelines might consider adding brain LITT as a therapeutic option in patients with recurrent high-grade gliomas in or near areas of eloquence. Lastly, a well-designed trial comparing brain LITT versus craniotomy in high-grade gliomas is warranted.

\section{References}

1 Chang EF, Smith JS, Change SM, Lamborn KR, Prados MD, Butowski N, et al: Preoperative prognostic classification system for hemispheric low-grade gliomas in adults. J Neurosurg 2008;109:817-824.

2 Ostrom QT, Gittleman H, Liao P, Rouse C, Chen Y, Dowling J, et al: CBTRUS statistical report: primary brain and central nervous system tumors diagnosed in the United States in 2007-2011. Neuro Oncol 2014;16:iv1iv63.

3 Lacroix M, Abi-Said D, Fourney DR, Gokaslan AL, Shu W, DeMonte F, et al: A multivariate analysis of 416 patients with glioblastoma multiforme: prognosis, extent of resection, and survival. J Neuorsurg 2001;95: 190-198.

4 McGirt MJ, Mukherjee D, Chaichana KL, Than KD, Quiones-Hinojosa A: Association of surgically acquired motor and language deficits on overall survival after resection of glioblastoma multiforme. Neurosurgery 2009;65:463-470.

5 Sanai N, Polley M-Y, McDermott MW, Parsa AT, Berger MS: An extent of resection threshold for newly diagnosed glioblastomas. J Neurosurg 2011;115:3-8.

6 Oszvald Á, Güresir E, Setzer M, Vatter H, Senft C, Seifert V, et al: Glioblastoma therapy in the elderly and the importance of extent of resection regardless of age. J Neurosurg 2012; 116:357-364.

7 Orringer D, Lau D, Khatri S, Zamora-Berridi GJ, Zhang K, Wu C, et al: Extent of resection in patients with glioblastoma: limiting factors, perception of resectability, and effect on survival. J Neurosurg 2012;117:851-859.
8 Hardesty DA, Sanai N: The value of glioma extent of resection in the modern neurosurgical era. Front Neurol 2012;3:140.

9 Gulati S, Jakola AS, Nerland US, Weber C, Solheim O: The risk of getting worse: surgically acquired deficits, perioperative complications, and functional outcomes after primary resection of glioblastoma. World Neurosurg 2011;76:572-579.

10 Konglund A, Helseth R, Lund-Johansen M, Helseth E, Meling TR: Surgery for high-grade gliomas in the aging. Acta Neurol Scand 2013; 128:185-193.

11 Sawaya R, Hammoud M, Schoppa D, Hess KR, Wu SZ, Shi WM, et al: Neurosurgical outcomes in a modern series of 400 craniotomies for treatment of parenchymal tumors. Neurosurgery 1998;42:1044-1055.

12 Jackson RJ, Fuller GN, Abi-Said D, Lang FF, Gokaslan ZL, Shi WM, et al: Limitations of stereotractic biopsy in the initial management of gliomas. Neuro Oncol 2001;3:193-200.

13 National Comprehensive Cancer Network Clinical Practice Guidelines in Oncology, Central Nervous System Cancers. Version 2. 2014. http://www.nccn.org/professionals/ physician_gls/f_guidelines.asp\#site (accessed June 16, 2015).

14 Park JK, Hodges T, Arko L, Shen M, Dello Iacono $\mathrm{D}, \mathrm{McNabb} \mathrm{A}$, et al: Scale to predict survival after surgery for recurrent glioblastoma multiforme. J Clin Oncol 2010;28:38383843 .

15 The Ottawa Hospital Research Institute. http://www.prisma-statement.org/statement. htm (accessed July 25, 2015).
16 Hozo SP, Djulbegovic B, Hoao I: Estimating the mean and variance from the median, range, and the size of the sample. BMC Med Res Methodol 2005;5:13.

17 Ascher PW: Interstitial thermal therapy of brain tumors with Nd:YAG laser under real time MRI control. Proc SPIE 1990;1200:242246.

18 Sakai T, Fujishima I, Sugiyama K, Ryu H, Uemura K: Interstitial laserthermia in neurosurgery. J Clin Laser Med Surg 1992;10:37-40.

19 Bettag M, Ulrich F, Schober R, Sabel M, Kahn $\mathrm{T}$, Hessel S, et al: Laser-induced interstitial thermotherapy of malignant gliomas. Adv Neurosurg 1992;20:253-257.

20 Reimer P, Bremer D, Horch C, Morgenroth C, Allkemper T, Schuierer G: MR-monitored LITT as a palliative concept in patients with a high grade glioma: preliminary clinical experience. J Magn Reson Imaging 1998;8:240244.

21 Schwarzmaier H-J, Eickmeyer F, von Tempelhoff W, Fiedler VU, Niehoff H, Ulrich SD, et al: MR-guided laser irradiation of recurrent glioblastomas. J Magn Reson Imaging 2005; 22:799-803.

22 Schwarzmaier H-J, Eickmeyer F, von Tempelhoff W, Fiedler VU, Neihoff H, Ulrich SD, et al: MR-guided laser-induced interstitial thermotherapy of recurrent glioblastoma multiforme: preliminary results in 16 patients. Eur J Radiol 2006;59:208-215.

23 Carpentier A, McNichols RJ, Itzcovitz J, Guichard J-P, Reizine D, Delaloge S, et al: Real-time magnetic resonance-guided laser thermal therapy for focal metastatic brain tumors. Neurosurgery 2008;63(suppl 1):ONS21-ONS29. 
24 Carpentier A, McNichols RJ, Stafford RJ, Guichard J-P, Reizine D, Delaloge S, et al: Laser thermal therapy: real-time MRI-guided and computer-controlled procedures for metastatic brain tumors. Lasers Surg Med 2011;43:943-950.

25 Carpentier A, Chauvet D, Reina V, Beccaria $\mathrm{K}$, Leclerq D, McNichols RJ, et al: MR-guided laser-induced thermal therapy (LITT) for recurrent glioblastomas. Lasers Surg Med 2012; 44:361-368.

26 Jethwa PR, Barrese JC, Gowda A, Shetty A, Danish SF: Magnetic resonance thermometry-guided laser-induced thermal therapy for intracranial neoplasms: initial experience. Neurosurgery 2012;71(1 Suppl Operative):133-145.

27 Sloan AE, Ahluwalia MS, Valerio-Pascua J, Mahnila S, Torchia MG, Phillips M, et al: Results of the NeuroBlate system first-in-humans phase 1 clinical trial for recurrent glioblastoma. J Neurosurg 2013;118:1202-1219.

28 Torres-Reveron J, Tomasiewicz HC, Shetty A, Amankulor NM, Chiang VL: Stereotactic laser induced thermotherapy (LITT): a novel treatment for brain lesions regrowing after radiosurgery. J Neurooncol 2013;113:495-503.

29 Schroeder JL, Missios S, Barnett GH, Mohammadi AM: Laser interstitial thermal therapy as a novel treatment modality for brain tumors in the thalamus and basal ganglia. Photonics Lasers Med 2014;3:151-158.

30 Mohammadi AM, Hawasli AH, Rodriguez A, Schroeder JL, Laxton AW, Elson P, et al: The role of laser interstitial thermal therapy in enhancing progression free survival of difficultto-access high-grad gliomas: a multicenter study. Cancer Med 2014;3:971-979.

31 Hawasli AH, Bagade S, Shimony JS, MillerThomas M, Leuthardt EC: Magnetic resonance imaging-guided focused laser interstitial thermal therapy for intracranial lesions: single-institution series. Neurosurgery 2013; 73:1007-1017.
32 Danks RA, Aglio LS, Gugino LD, Black PM: Craniotomy under local anesthesia and monitored conscious sedation for the resection of tumors involving eloquent cortex. J Neurooncol 2000;49:131-139.

33 Kim SS, McCutcheon IE, Suki D, Weinberg JS, Sawaya R, Lang FF, et al: Awake craniotomy for brain tumors near eloquent cortex: correlation of intraoperative cortical mapping with neurological outcomes in 309 consecutive cases. Neurosurgery 2009;64:836846.

34 Sanai N, Polley M-Y, Berger MS: Insular glioma resection: assessment of patient morbidity, survival, and tumor progression. J Neurosurg 2010;112:1-9.

35 Kuhnt D, Becker A, Ganslandt O, Bauer M, Buchfelder M, Nimsky C: Correlation of the extent of tumor volume resection and patient survival in surgery of glioblastoma multiforme with high-field intraoperative MRI guidance. Neuro Oncol 2011;13:1339-1348.

36 Kreig SM, Schnurbus L, Shiban E, Droese D, Obermueller T, Buchmann N, et al: Surgery of highly eloquent gliomas primarily assessed as non-resectable: risks and benefits in a cohort study. BMC Cancer 2013;12:51.

37 Bloch O, Han SJ, Cha S, Sun MZ, Aghi MK, McDermott MW, et al: Impact of extent of resection for recurrent glioblastoma on overall survival. J Neurosurg 2012;117:1032-1038.

38 Satoer D, Vork J, Visch-Brink E, Smits M, Dirven C, Vincent A: Cognitive functioning early after surgery of gliomas in eloquent areas. J Neurosurg 2012;118:831-838.

39 Chaichana KL, Jusue-Torres I, NavarroRamirez R, Raza SM, Pascual-Gallego M, Ibrahim A, et al: Establishing percent resection and residual volume thresholds affecting survival and recurrence for patients with newly diagnosed intracranial glioblastoma. Neuro Oncol 2014;16:113-122.
40 Hundsberger T, Tonder M, Hottinger A, Brügge D, Roekche U, Putora PM, et al: Clinical management and outcome of histologically verified adult brain stem gliomas in Switzerland: a retrospective analysis of 21 patients. J Neurooncol 2014;118:321-328.

41 Schucht P, Seidel K, Beck J, Murek M, Jilch A, Wiest $\mathrm{R}$, et al: Intraoperative monopolar mapping during 5-ALA-guided resections of glioblastomas adjacent to motor eloquent areas: evaluation of resection rates and neurological outcome. Neurosurg Focus 2014;37: $1-8$.

42 Della Puppa A, Ciccarino P, Lombardi G, Rolma G, Cecchin D, Rossetto M: 5-Aminolevulinic acid fluorescence in high grade glioma surgery: surgical outcome, intraoperative findings, and fluorescence patterns. Biomed Res Int 2014;2014:232561.

43 Renovanz M, Hickmann A-K, Henkel C, Nadji-Ohl M, Hopf NJ: Navigated versus non-navigated intraoperative ultrasound: is there any impact on the extent of resection of high-grade gliomas? A retrospective clinical analysis. J Neurol Surg 2014;75:224-230.

44 Schucht P, Beck J, Seidel K, Raabe A: Extending resection and preserving function: modern concepts of glioma surgery. Swiss Med Wkly 2015;145:w14082.

45 De Witt Hamer PC, Robles SG, Zwinderman AH, Duffau H, Berger MS: Impact of intraoperative stimulation brain mapping on glioma surgery outcome: a meta-analysis. J Clin Oncol 2012;30:2559-2565.

46 Weller M, Cloughesy T, Perry JR, Wick W: Standards of care for treatment of recurrent glioblastoma - are we there yet? Neuro Oncol 2013;15:4-27.

47 Munireddy S, Katz S, Somasundar P, Espat NJ: Thermal tumor ablation therapy for colorectal cancer hepatic metastasis. J Gastrointest Oncol 2012;3:69-77.

48 Xu Q, Kobayashi S, Ye X, Meng X: Comparison of hepatic resection and radiofrequency ablation for small hepatocellular carcinoma: a meta-analysis of 16,103 patients. Sci Rep 2014;4:7252. 